\title{
Aging in Fully Annealed and Subsequently Strained Poly(methyl methacrylate)
}

\author{
Yukuo NANZaI, ${ }^{\dagger}$ Akihiro Miwa, and Shan Zi CUI \\ Department of Mechanical Engineering, Osaka City University, \\ Osaka 558-8585, Japan
}

(Received May 31, 1999)

\begin{abstract}
Aging in fully annealed and subsequently strained poly(methyl methacrylate) (PMMA) was studied by mechanical compression, DSC and thermally stimulated deformation recovery. Evolution of yield stress with aging was observed for compressively strained and aged PMMA specimens. Evolution of yield stress was much more significant than that in quenched and physically aged PMMA. Experimental data obtained by DSC and the thermally stimulated deformation recovery indicated strain energy given to the annealed PMMA specimen to be locked into physically aged structure more tightly with aging time. Thus, the evolution of yield stress of annealed and strained PMMA is probably due to the physically aged structure locking the strain energy tightly in itself. KEY WORDS Aging / Poly(methyl methacrylate) / Compressive Strain / Yield Stress / Strain Energy / Differential Scanning Calorimetry / Deformation Recovery /
\end{abstract}

Amorphous polymeric material heated at a temperature above the glass transition temperature $T_{\mathrm{g}}$ comes to a metastable glassy state if slowly cooled through $T_{\mathrm{g}}$. After heat treatment, mechanical properties of this metastable structure are expected to be almost unchanged with time. However, if the metastable amorphous polymer is subjected to a finite stress or strain as seen in tight fastening by bolts and nuts, the structural stability of the polymer cannot be the case. As illustrated previously, ${ }^{1-5}$ the metastable structure of an amorphous polymer is changed to an unstable one by the imposition of stress or strain, and mechanical properties change with time. As a specific case showing this structural change, we reported that dynamic viscoelastic properties of poly(methyl methacrylate) (PMMA) tensioned by a strain of 0.1 at a temperature of $80^{\circ} \mathrm{C}$ changed with time and at long times the storage modulus reached a value much higher than that of the unstrained metastable sample. ${ }^{3}$ This is aging behavior apparently similar to that observed in quenched amorphous polymers referred to as physical aging. ${ }^{6}$ Evolution of modulus far beyond that in the unstrained metastable state cannot easily result from aging in quenched polymers because the metastable state is a goal of the aging process in quenched polymers. Thus, aging in strained metastable amorphous polymers is supposedly ascribable to a mechanism different from that in quenched polymers. Yet, aging in strained polymers has not substantially been studied in spite of its practical significance in engineering applications of polymers.

The present study examines aging in fully annealed and then strained PMMA using quite slowly cooled samples by means of a mechanical test, a differential scanning calorimetry (DSC) and thermally stimulated deformation recovery.

\section{EXPERIMENTAL}

A commercial cast sheet of PMMA was used as the experimental material. The glass transition temperature

\footnotetext{
+ To whom correspondence should be addressed.
}

of the sample was determined as $116^{\circ} \mathrm{C}$ by DSC run at a heating rate of $1 \mathrm{~K} \mathrm{~min}^{-1}$. Using gel permeation chromatography, the molecular weight $M_{n}$ and polydispersity $M_{w} / M_{n}$ of the sample were evaluated as $7.2 \times 10^{5}$ and 4.7 , respectively. Cylindrical specimens $5 \mathrm{~mm}$ in diameter and $10 \mathrm{~mm}$ in height were cut from the sample sheet for mechanical and deformation recovery tests. For DSC measurement, smaller cylindrical specimens $3.5 \mathrm{~mm}$ in diameter and $5 \mathrm{~mm}$ in height were cut from the same sample sheet. These small specimens could directly be put into a DSC capsule without further processing after aging.

After being heated at $130^{\circ} \mathrm{C}$ for $2 \mathrm{~h}$, cylindrical specimens of both sizes were cooled from $130^{\circ} \mathrm{C}$ to $90^{\circ} \mathrm{C}$ at a quite slow rate of $3 \mathrm{~K} \mathrm{~h}^{-1}$ and then from $90^{\circ} \mathrm{C}$ to room temperature at $10 \mathrm{~K} \mathrm{~h}^{-1}$. These fully annealed specimens are hereafter designated as thermally conditioned specimens $A$ or simply as specimens $A$. In contrast to specimen $\mathrm{A}$, we prepared quenched specimens by blowing air of room temperature onto the specimens heated at $130^{\circ} \mathrm{C}$ to compare aging behavior with that in specimen $\mathrm{A}$. The quenched specimens will be designated as thermally conditioned specimens $Q$ or simply as specimens Q.

One specimen $\mathrm{A}$ of diameter $5 \mathrm{~mm}$ was put into a temperature controlled cabinet kept at a constant temperature of $90^{\circ} \mathrm{C}$ and left there for $3 \mathrm{~min}$ to achieve thermal equilibrium. Then we compressed the specimen at a strain rate of $\dot{\varepsilon}=8.8 \times 10^{-4} \mathrm{~s}^{-1}$ up to a strain $\varepsilon_{\mathrm{a}}$ of either 0.04 or 0.08 , and stopped the compression machine to leave the specimen in the strained condition. The specimen was aged at $90^{\circ} \mathrm{C}$ for time periods from $2 \mathrm{~min}$ to $24 \mathrm{~h}$ in the strained state. During the aging, the stress in the specimen relaxed with time. After a fixed time period of aging, we resumed the constant rate compression of the specimen at $\dot{\varepsilon}$ to obtain the stress-strain relation of the strained and aged specimen. True stress $\sigma$ and true strain $\varepsilon$ were calculated from a load-deformation curve based on the assumption of isovolume and uniform deformation of the specimen.

A thermally conditioned specimen A of $3.5 \mathrm{~mm}$ in diameter was first aged in the strained state for $2 \mathrm{~min}$ to 
$24 \mathrm{~h}$ and then quickly cooled to room temperature by a steady blow of air to preserve the aged structure of the specimen in a frozen state at room temperature. This strained and then aged specimen $\mathrm{A}$ was subjected to DSC measurement using a DSC 6100 calorimeter of Seiko Instruments run at a heating rate of $1 \mathrm{~K} \mathrm{~min}^{-1}$ between $-30^{\circ} \mathrm{C}$ and $150^{\circ} \mathrm{C}$.

Thermally stimulated deformation recovery was measured on strained and then aged specimens A of diameter $5 \mathrm{~mm}$. After aging, the specimen was quickly cooled by a steady blow of air and heated at a rate of $1 \mathrm{~K} \mathrm{~min}^{-1}$ from room temperature to $150^{\circ} \mathrm{C}$. Deformation recovery of the specimen was detected by a laser displacement detector produced by Keyence Co.

Thermally conditioned specimens $Q$ were exposed to a temperature of $90^{\circ} \mathrm{C}$ for $3 \mathrm{~min}$ to achieve thermal equilibrium and aged for durations from $1 \mathrm{~h}$ to $2000 \mathrm{~h}$ at $90^{\circ} \mathrm{C}$. They were then quickly moved to a temperature controlled cabinet of the compression machine, and compressed at $\dot{\varepsilon}$.

DSC measurement for the aged specimen $Q$ was made on the specimens quickly cooled by air to room temperature at the end of aging at $90^{\circ} \mathrm{C}$.

\section{RESULTS}

Specimens A strained by 0.04 in compression were aged in the strained state at $90^{\circ} \mathrm{C}$ for various periods up to $24 \mathrm{~h}$. The compressive true stress-strain $(\sigma-\varepsilon)$ relations of such strained and aged specimens $A$ were obtained at $90^{\circ} \mathrm{C}$ as shown in Figure 1. In the figure, the dashed curve plotted against the logarithm of aging time $t_{\mathrm{a}}$ gives the stress relaxation behavior after straining up to $\varepsilon_{\mathrm{a}}=0.04$. At the end of aging time $t_{\mathrm{a}}$, constant rate compression of the aged specimen was continued. In Figure 1, the stress-strain relations of aged specimens $A$ are drawn as solid curves rising linearly from the dashed line and horizontally shifted by $\log t_{\mathrm{a}}$ along the axis of $\varepsilon$. A solid curve drawn from the origin of the axes $\sigma$ and $\varepsilon$ gives the stress-strain relation for the thermally conditioned specimen A, showing that the strain of 0.04 is little larger than that at the limit of initial linear region of $\sigma-\varepsilon$ curve, yet significantly less than the yield strain. As seen in Figure 1, the yield stress of specimens A provided with the strain of 0.04 increased continuously with aging time.

The stress-strain relations of specimens A with strain of 0.08 are shown in Figure 2. As seen from the $\sigma-\varepsilon$ relation of the thermally conditioned specimen A (solid curve drawn from the origin), the strain has exceeded that of the yield point. The stress relaxation in the strained state shown by a dashed line is much faster than that in the case of $\varepsilon_{\mathrm{a}}=0.04$ as shown by the dashed line in Figure 1. This indicates that the metastable structure has changed into a softened liquid-like structure. $^{7}$ Due to this softened structure, the yield stress at the resumed compression after a comparatively short time of aging was lower than that of unaged samples. However, at long times of aging the yield stress evolved to exceed that of the unaged sample.

Time evolution of yield stress similar to those shown in Figures 1 and 2 has generally been found also in quenched amorphous polymers, and is referred to as

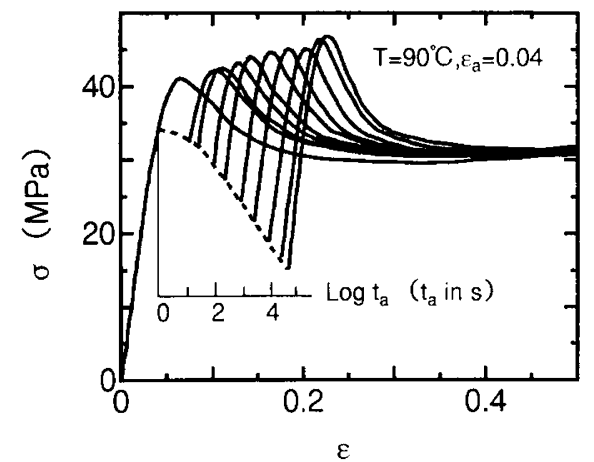

Figure 1. Compressive true stress-strain relations for poly(methyl methacrylate) (PMMA) specimens annealed and aged under compressive strain of 0.04 , showing evolution of yield stress with increasing aging time $t_{\mathrm{a}}$.

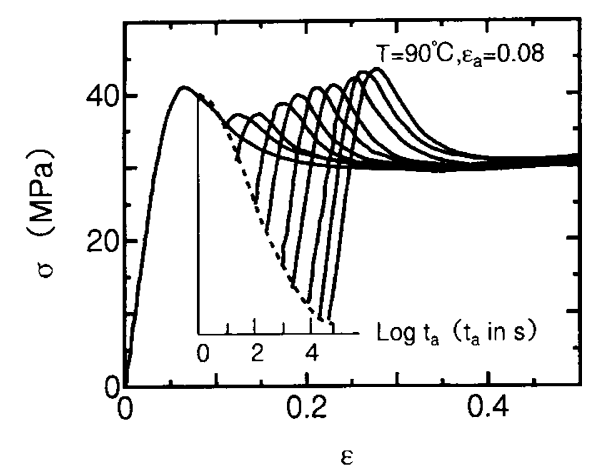

Figure 2. Evolution of yield stress with aging time $t_{\mathrm{a}}$ in PMMA specimens annealed and aged under compressive strain of 0.08 .

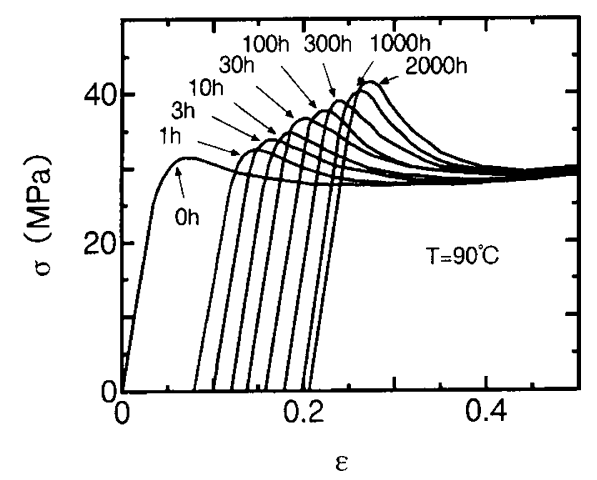

Figure 3. Variation of compressive true stress-strain relations with aging time for quenched PMMA specimens.

physical aging. ${ }^{6}$ To compare the physical aging in the same PMMA sample with aging in the strained samples, we measured the time evolution of yield stress for quenched samples (specimens Q). The change of compressive stress-strain relation with time for specimens Q aged from 1 to $2000 \mathrm{~h}$ at $90^{\circ} \mathrm{C}$ is shown in Figure 3 , where each $\sigma-\varepsilon$ curve has horizontally shifted along the $\varepsilon$ axis nearly in proportion to the logarithm of aging time.

To compare time evolutions of yield stress $\sigma_{\mathrm{y}}$ separately shown in Figures 1, 2, and 3, they are summarized in Figure 4 as a plot of $\sigma_{\mathrm{y}}$ against $\log t_{\mathrm{a}}$. A horizontal dash-dot line in the figure gives the magnitude of the yield stress for the thermally conditioned specimen A, while a dotted line near the bottom shows the value of the yield stress of the thermally conditioned specimen 


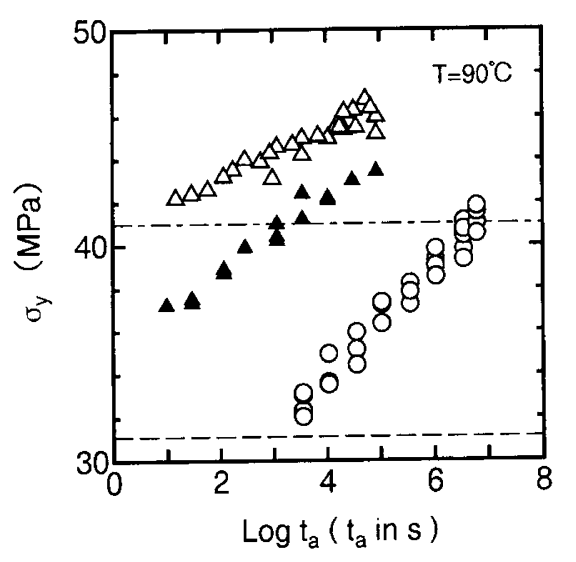

Figure 4. Yield stress $\sigma_{y}$ plotted against the logarithm of aging time $t_{\mathrm{a}}$ for three aged PMMA specimens. $\triangle$, annealed specimens aged under a strain of $0.04 ; \boldsymbol{\Delta}$, annealed specimens aged under a strain of 0.08 ; $\bigcirc$, quenched and aged specimens.

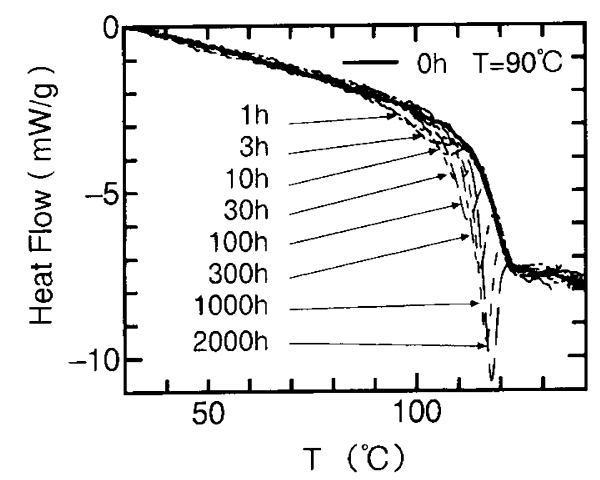

Figure 5. Change in differential scanning calorimetry (DSC) thermograms with aging time for quenched PMMA.

Q. The mean value of yield stress obtained at the aging time of $2000 \mathrm{~h}$ (the longest aging time for specimens $\mathrm{Q}$ in the present experiment) is almost consistent with the stress level of the dash-dot line. This indicates that the structure aged for $2000 \mathrm{~h}$ in the quenched sample is presumably identical with a metastable structure in the thermally conditioned specimen $\mathrm{A}$ resulted through the quite slow cooling. As shown in Figure 4, the yield stress of specimens A strained by either 0.04 or 0.08 and then aged increased linearly with $\log t_{\mathrm{a}}$. In the case of $\varepsilon_{\mathrm{a}}=0.04$, the yield stress increased more than $10 \%$ of that for the thermally conditioned specimen $\mathrm{A}$ within an aging time of $24 \mathrm{~h}$.

As seen in Figure 4, three curves for aged specimens of the different histories seem to form a continuous master curve if they are provided with appropriate horizontal shifts. This master curve, hence, permits one to expect that the evolution of yield stress of specimens $\mathrm{Q}$ at aging times longer than $2000 \mathrm{~h}$ possibly continues following the evolution curves for strained and then aged specimens $A$. That is, one may simply suppose that the aging in specimens $Q$ is accelerated by the imposition of strain. This conjecture led us to the DSC examination of the strained and then aged specimens $\mathrm{A}$ and the aged specimens $\mathrm{Q}$.

Differential scanning calorimetry thermograms for specimens $Q$ at various aging times are plotted against temperature in Figure 5. An endothermic peak is seen

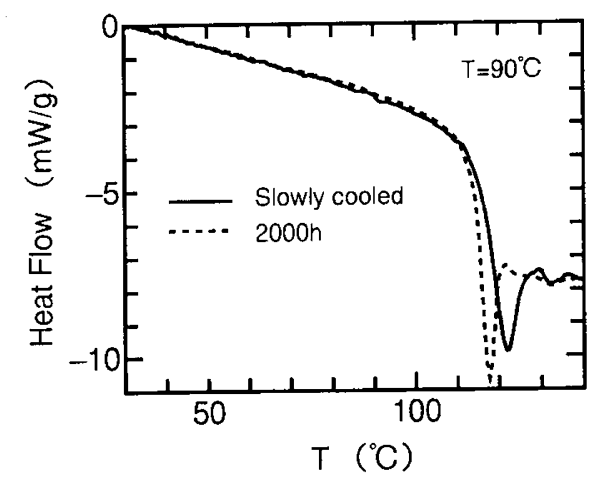

Figure 6. DSC thermograms for slowly cooled (annealed) PMMA specimen compared with that for quenched specimens aged for $2000 \mathrm{~h}$.

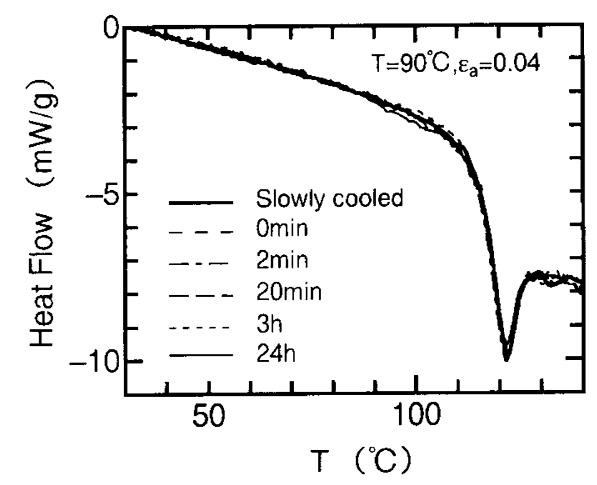

Figure 7. Variation of DSC thermograms with aging time for PMMA annealed and aged under strain of 0.04 .

to move to higher temperatures with increasing aging time. This is an evolution of the endothermic peak generally known in quenched amorphous polymers. ${ }^{6}$ In Figure 6, the DSC thermogram obtained at $2000 \mathrm{~h}$ was compared with that for thermally conditioned specimen A. The endothermic peak for specimen A appeared at $5 \mathrm{~K}$ higher than that for the aged specimen $\mathrm{Q}$. Hence, the temperature difference between these peaks indicates that the polymeric structure in the thermally conditioned specimen $\mathrm{A}$ is thermally more stable than that in the specimen $Q$ aged for $2000 \mathrm{~h}$, although the yield stress of these specimens was quite similar as seen in Figure 4. Yield stress for such fairly stable structure may thus be less dependent on thermal structural stability in comparison to that for an unstable structure.

In Figures 7 and 8, DSC thermograms of the strained specimens A are shown for $\varepsilon_{\mathrm{a}}=0.04$ and $\varepsilon_{\mathrm{a}}=0.08$, respectively. In Figure 7 , comparing the data with a thermogram for unaged specimen (a bold line), we can see a weak and broad endothermic behavior for a specimen of aging time $t_{\mathrm{a}}=24 \mathrm{~h}$. At less aging time, however, we could not find significant difference between the DSC thermograms of the aged and unaged specimens in spite of the distinct increase in yield stress as seen in Figure 4. In Figure 8, endothermic peaks at temperatures below $T_{\mathrm{g}}$ are distinguishable for specimens of $t_{\mathrm{a}}=3 \mathrm{~h}$ and $24 \mathrm{~h}$. The details of these peaks are shown enlarged in Figure 9, showing that the peak evolves with increasing aging time. In Figure 8, an endothermic peak appearing at a temperature above $T_{\mathrm{g}}$ is attenuated with increasing aging time as reproduced distinctly on an 


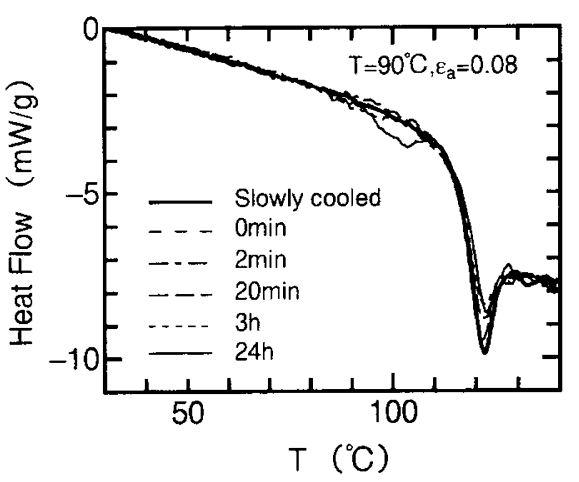

Figure 8. Variation of DSC thermograms with aging time for PMMA annealed and aged under strain of 0.08 .

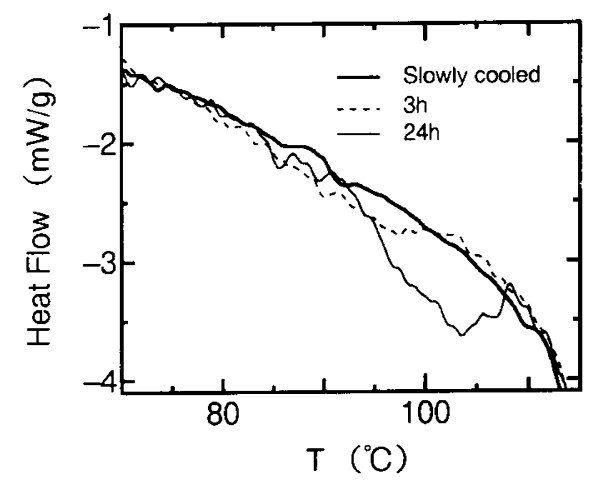

Figure 9. Details of variation in DSC thermograms with aging time at temperatures below $T_{\mathrm{g}}$ for PMMA annealed and aged under the strain of 0.08 .

enlarged scale in Figure 10. Accordingly, comparing Figures 5, 7, and 8, we can conclude that change in DSC thermograms with aging time for strained specimens A is qualitatively different from that for specimens $Q$. In other words, the DSC thermograms obtained for strained specimens A probably indicates the aging in strained specimens A not ascribable to the acceleration of the aging in specimens $Q$, but to be controlled by a mechanism essentially different from that of the aging in quenched amorphous polymers.

\section{DISCUSSION}

If a metastable amorphous polymeric structure such as that of the thermally conditioned specimen $\mathrm{A}$ is given a finite mechanical strain, the structure changes to non-equilibrium. ${ }^{1-5}$ After imposition of the finite strain, the stress relaxes gradually and the non-equilibrium structure changes with time towards a certain quasiequilibrium structure. In this relaxation, structural change similar to that observed in quenched amorphous polymers, i.e., ordinary physical aging process, may occur because in Figure 8 the evolution of endothermic peak with time in the sub- $T_{\mathrm{g}}$ range is similar to that observed for quenched polymers. From a comparison of Figures 5 , 7, and 8 , there is no simple similarity in DSC thermograms between strained specimens $A$ and the specimens $Q$. Thus we conclude that an aging mechanism essentially different from that in quenched polymers is probably effective. To analyze the complicated situation

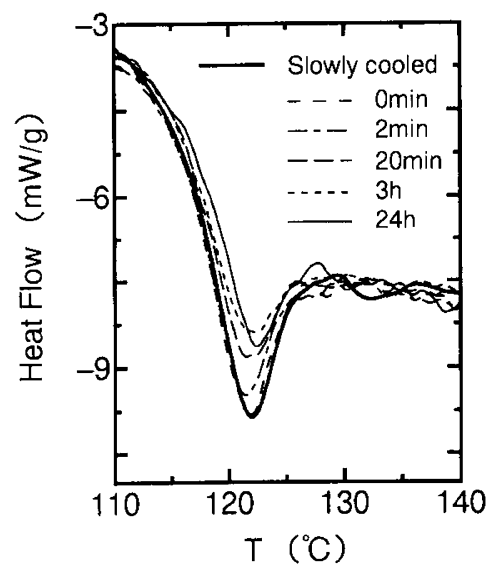

Figure 10. Details of variation in DSC thermograms with aging time at temperatures above $T_{\mathrm{g}}$ for PMMA annealed and aged under strain of 0.08 .

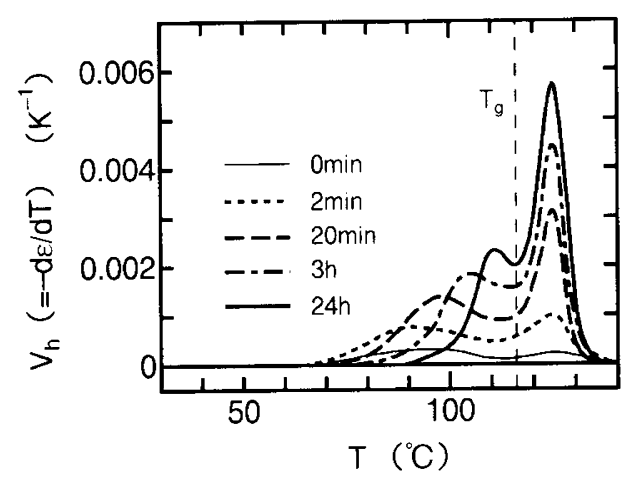

Figure 11. Variation with aging time in plots of strain recovery rate against temperature $T$ for PMMA annealed and aged under the strain of 0.08 .

of this aging in the strained specimens $\mathrm{A}$, we measured thermally stimulated deformation recovery of the strained and then aged specimens $A$. This is because the deformation recovery reveals qualitatively the state of strain energy stored in strained samples.

In Figure 11, thermally stimulated deformation recovery for strained and aged specimens $\mathrm{A}$ is plotted as strain recovery rate $(\mathrm{d} \varepsilon / \mathrm{d} T)$ against temperature. The specimens were strained by 0.08 and then aged variously for $2 \mathrm{~min}$ to $24 \mathrm{~h}$. As seen in the figure, there appears two recovery peaks at the constant rate of heating: one is at a temperature below $T_{\mathrm{g}}$ and another is above $T_{\mathrm{g}}$. The area under each curve gives residual plastic strain after aging, and this area increases with aging time, because the residual plastic strain increases with aging time due to the stress relaxation, i.e., decrease in stress effective for elastic deformation recovery after unloading. Concerning the recovery peak below $T_{\mathrm{g}}$, Oleynik $^{8}$ reported that in a temperature range around this peak some amount of strain energy stored in the sample is released. We thus must take into account this energy release (i.e., exothermic effect) when examining DSC results such as shown in Figure 9. Let us re-examine the DSC thermograms of Figure 9 by comparing them closely with deformation recovery shown in Figure 11. The plot for comparison is shown in Figure 12. Arrow 1 on the top panel points to the peak of sub- $T_{\mathrm{g}}$ deformation recovery in the case 

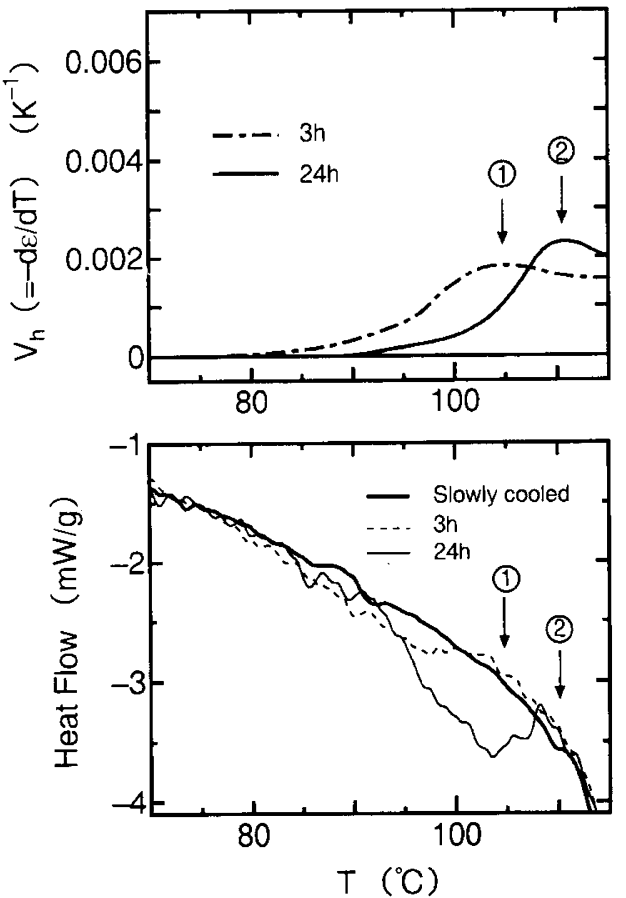

Figure 12. Deformation recovery curves (top panel) closely compared to DSC thermograms (bottom panel) for PMMA annealed and aged under strain of 0.08 .

of $t_{\mathrm{a}}=3 \mathrm{~h}$. The strain energy is expected to have been released around the temperature pointed by the arrow 1. In the same temperature range of the arrow 1 on the bottom panel, the endothermic curve is depressed towards the exothermic direction, probably because of the thermal energy balance between the endothermic behavior caused by an aged structure brought by ordinary physical aging and the exothermic effect due to the release of strain energy. Similar depression of the endothermic behavior is seen also in the case of $t_{\mathrm{a}}=24 \mathrm{~h}$ around the temperature pointed by arrow 2 . Endothermic behavior due to the structure brought by ordinary physical aging began to appear first, and then at slightly higher temperatures (around arrow 2) strain energy was presumably released simultaneously with the endothermic behavior. Thus, a transient part of the thermogram is first endothermic and then depressed towards the exothermic direction, indicating that the strain energy has been locked into the molecular structure stabilized by ordinary physical aging. That is, the strain energy could be released after the stabilized structure locking that strain energy in itself had been loosened by the thermal energy absorption.

The steady shift of the sub- $T_{\mathrm{g}}$ recovery peak to higher temperatures with aging time indicates that the strain energy comes to be locked in the stabilized structure more tightly with increasing aging time. Since the yield stress increased simultaneously with shift of the sub- $T_{\mathrm{g}}$ recovery peak to higher temperatures, we can conclude that the stabilized structure locking the strain energy in itself contributes to the significant increase in yield stress. The increase in yield stress with aging time reminds us of the solid solution strengthening in metallic alloys although they are definitely different from amorphous polymers in structure: In metallic alloys, the excess strain energy induced by diffusion of solution atoms into the matrix metallic lattice is effective to increase the yield stress of alloys. ${ }^{9}$ However, at present we are not yet able to interpret thoroughly the mechanism of increase in yield stress of strained and aged polymers.

As shown in Figure 10, the endothermic peak at about $122^{\circ} \mathrm{C}$ was attenuated with aging time. At long times, if a part of the strain energy in the stabilized structure is locked so tightly as not to be released until at a temperature above $T_{\mathrm{g}}$, then only above this temperature the locked strain energy is released. Under such a condition, the energy release occurs simultaneously with endothermic behavior above $T_{\mathrm{g}}$, and thus apparently lessens the height of endothermic peak. The idea of such strain energy locked quite tightly into the aged structure allows us to mention that the endothermic peak attenuated with aging time probably results from aging in strained polymeric materials.

As seen in Figure 11, there appeared a deformation recovery peak at about $125^{\circ} \mathrm{C}$. The peak is due to the $\alpha$ motion of polymer chains thermally stimulated at this temperature. ${ }^{6}$ In Figure 10, the DSC endothermic peak appeared at about $122^{\circ} \mathrm{C}$, which is a little lower than the temperature of the deformation recovery peak. This temperature difference presumably indicates such that, at temperatures above $T_{\mathrm{g}}$ the metastable structure is first loosened by thermal stimulation and nearly at the same time the strain energy having quite tightly been locked is released. The $\alpha$ motion of polymer chains is allowed and the peak of deformation recovery is observed at the temperature a little higher than that of endothermic peak.

As for the deformation recovery in the case of $\varepsilon_{\mathrm{a}}=0.04$, strain remaining even after the aging time of $24 \mathrm{~h}$ was so small that we could not obtain well-defined recovery behavior such as shown in Figure 11. As seen in Figure 7 , in the case of $\varepsilon_{\mathrm{a}}=0.04$, we had only weak and broad endothermic behavior for the specimen of $t_{\mathrm{a}}=24 \mathrm{~h}$. At present, we are not able to discuss precisely the structures of strained and aged specimens $A$ for $\varepsilon_{\mathrm{a}}=0.04$. Yet, supposedly similar to the aged structure in the case of $\varepsilon_{\mathrm{a}}=0.08$, the evolution of yield stress for $\varepsilon_{\mathrm{a}}=0.04$ is caused by structure once disordered by strain of 0.04 and then aged simultaneously with the locking of strain energy into itself.

\section{CONCLUSIONS}

Significant evolution of yield stress with aging time was noted for fully annealed and then strained poly(methyl methacrylate). Experimental data obtained by the DSC and thermally stimulated deformation recovery shows that the strain energy given to the annealed PMMA specimen is locked in physically aged structure more tightly with aging time. Thus, the evolution of yield stress in annealed and strained PMMA is probably due to physically aged structure locking the strain energy tightly in itself.

\section{REFERENCES}

1. Y. Nanzai, Polym. Eng. Sci., 30, 96 (1990).

2. Y. Nanzai, T. Konishi, and S. Ueda, J. Mater. Sci., 26, 4477 (1991). 
Y. NANZal, A. Miwa, and S. Z. CuI

3. S. Yoshioka, H. Usada, and Y. Nanzai, J. Non-Cryst. Sol., 172-174, 765 (1994).

4. Y. Nanzai and N. Nakayama, J. Non-Cryst. Sol., 172-174, 771 (1994).

5. Y. Nanzai, T. Yamasaki, and S. Yoshioka, JSME Intl. J., 41, 31 (1998).
6. L. C. E. Struik, "Physical Aging in Amorphous Polymers and Other Materials," Elsevier, Amsterdam, 1978.

7. Y. Nanzai, J. Non-Cryst. Sol., 131-133, 516 (1991).

8. E. Oleynik, Progr. Colloid Polym. Sci., 80, 140 (1989).

9. T. H. Courtney, "Mechanical Behavior of Materials," McGrawHill, New York, N.Y., 1990. 